\title{
Case Report \\ Remission Achieved in Refractory Advanced Takayasu Arteritis Using Rituximab
}

\author{
D. Ernst, ${ }^{1}$ M. Greer, ${ }^{2}$ M. Stoll, ${ }^{1}$ D. Meyer-Olson, ${ }^{1}$ R. E. Schmidt, ${ }^{1}$ and T. Witte ${ }^{1}$ \\ ${ }^{1}$ Clinic of Immunology and Rheumatology, Medical School Hannover, Carl-Neuberg-Straße 1, 30625 Hannover, Germany \\ ${ }^{2}$ Department of Pneumology, Medical School Hannover, Carl-Neuberg-Straße 1, 30625 Hannover, Germany
}

Correspondence should be addressed to D. Ernst, ernst.diana@mh-hannover.de

Received 16 August 2012; Accepted 18 September 2012

Academic Editors: S. S. Koca, J. Mikdashi, P. Njobvu, and F. Schiavon

Copyright (C) 2012 D. Ernst et al. This is an open access article distributed under the Creative Commons Attribution License, which permits unrestricted use, distribution, and reproduction in any medium, provided the original work is properly cited.

\begin{abstract}
A 25-year-old patient was referred due to subclavian stenosis, identified on echocardiography. She presented with exertional dizziness and dyspnoea. Questioning revealed bilateral arm claudication. Examination demonstrated an absent right ulnar pulse and asymmetrical brachial blood pressure. Bruits were evident over both common carotid arteries. Doppler ultrasound and MRI angiograms revealed occlusion or stenosis in multiple large arteries. Takayasu arteritis (TA) was diagnosed and induction therapy commenced: $1 \mathrm{mg} / \mathrm{kg}$ oral prednisolone and $500 \mathrm{mg} / \mathrm{m}^{2}$ intravenous cyclophosphamide (CYC). Attempts to reduce prednisolone below $15 \mathrm{mg} / \mathrm{d}$ proved impossible due to recurring disease activity. Adjuvant azathioprine $100 \mathrm{mg} / \mathrm{d}$ was subsequently added. Several weeks later, the patient was admitted with a left homonymous hemianopia. The culprit lesion in the right carotid artery was surgically managed and the patient discharged on azathioprine $150 \mathrm{mg} / \mathrm{d}$ and prednisolone $30 \mathrm{mg} / \mathrm{d}$. Despite this, deteriorating exertional dyspnoea and angina pectoris were reported. Reimaging confirmed new stenosis in the right pulmonary artery. Surgical treatment proved infeasible. Given evidence of refractory disease activity on maximal standard therapy, we initiated rituximab, based on recently reported B-cell activity in TA.
\end{abstract}

\section{History}

A 25-year-old ICU nurse was referred for further assessment of a subclavian artery stenosis, recently diagnosed on echocardiography. This investigation was performed as part of cardiology assessment for recent-onset dizziness and dyspnoea with moderate exertion: $3 \mathrm{~km}$ cycling on level terrain. Heart catheterisation excluded relevant coronary artery disease. With exception of a successfully treated episode of optic neuritis in the right eye 7 years previously, there was no significant past medical history. Investigations at that time had focused on excluding multiple sclerosis, but a small right-sided parietal infarct was noted in the cerebral MRI. Following steroid treatment, the visual disturbance resolved completely. No further investigation or treatment regarding the infarct was performed at that time. The patient had never smoked and had not been taking any regular medication.

\section{Examination}

On general inspection, the patient appeared unremarkable; height: $164 \mathrm{~cm}$ and weight: $57.4 \mathrm{~kg}$. Significant clinical findings included an absent right ulnar pulse. Asymmetrical blood pressure measurements were noted: left $88 / 64 \mathrm{mmHg}$ and right $74 / 48 \mathrm{mmHg}$. Auscultation revealed a loud systolic murmur throughout the praecordium, along with bruits over the carotid, subclavian, and femoral arteries bilaterally and the left axillary artery. Skin and joint examinations were entirely unremarkable. There was no clinical suggestion for significant lymphadenopathy. Neurological examination was completely normal.

\section{Investigations}

Routine blood investigations revealed a microcytic anaemia along with raised ESR and CRP at $36 \mathrm{~mm} / \mathrm{h}$ and $45 \mathrm{mg} / \mathrm{L}$, respectively. Renal function was normal. The ANA titre was 


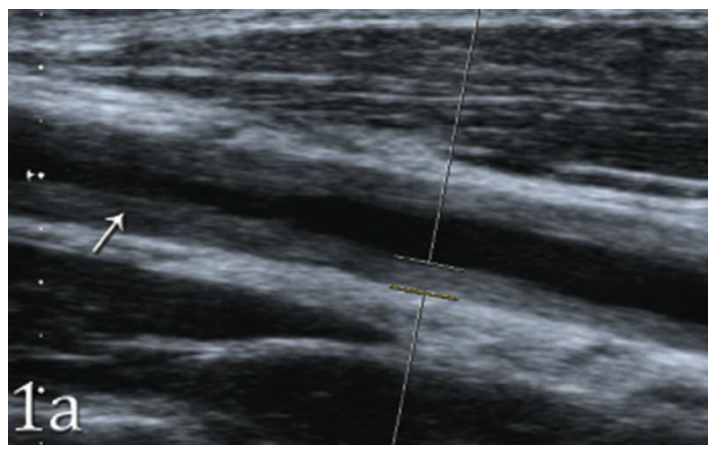

(a)

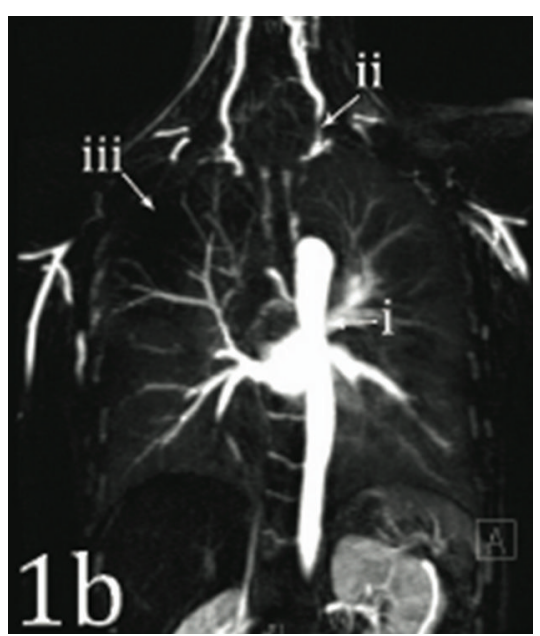

(b)

FIGURE 1: (a) Doppler ultrasound of the left common carotid artery demonstrating marked wall thickening with subsequent loss of lumen inkeeping with stenosis (arrow). (b) MR angiothorax/neck exhibiting widespread pathological changes in the arterial vessels: narrowing of the thoracic aorta (i), stenosis of the left carotid artery (ii), and bilateral occlusion of the subclavian arteries (iii), with collateral distal filling via the intercostal arteries.

minimally elevated at $1: 320$. Other immunological indices including ANCA and cryoglobulins were normal.

To further assess the degree and distribution of arterial stenoses, numerous Doppler ultrasound scans were performed. Carotid Dopplers revealed high-grade stenoses at the bifurcation of the left common carotid artery (Figure 1(a)) and medium grade at the bifurcation of the right common carotid artery. Further assessment demonstrated significant wall thickening in both subclavian arteries, with diminished flow on the left.

In the abdomen, a high-grade stenosis of the right renal artery was observed. In both dorsal pedal arteries, an occlusion pressure in excess of $200 \mathrm{mmHg}$ was recorded.

Given these findings, MR angiography of the head, neck, and thorax (Figure 1(b)) was performed, revealing both acute inflammation and extensive established disease: complete occlusion of the left subclavian and both axillary arteries with good collateralisation. Numerous short high-grade stenoses of the left common carotid artery with complete occlusion of the ipsilateral external carotid artery were seen. Minimal wall thickening with patchy contrast enhancement was observed in the thoracic aorta. A PET-CT scan was considered, but the patient declined this investigation due to the required radiation exposure.

\section{Treatment and Clinical Course}

Given that all ACR criteria for Takayasu arteritis were fulfilled [1], aggressive induction therapy in accordance with the current EULAR/EUVAS recommendations [2] was commenced: $1 \mathrm{mg} / \mathrm{kg}$ oral prednisolone (initially $60 \mathrm{mg}$, with subsequent tapering-see Figure 2). Due to the disappointing CRP response in the ensuing weeks, adjuvant treatment with intravenous cyclophosphamide in 4 weekly intervals was added: initially $500 \mathrm{mg} / \mathrm{m}^{2} ; 2$ nd dose
$750 \mathrm{mg} / \mathrm{m}^{2}$ with subsequent courses $1000 \mathrm{mg} / \mathrm{m}^{2}$. In total 6 cycles of cyclophosphamide were administered. Thereafter maintenance treatment with azathioprine $100 \mathrm{mg} / \mathrm{d}$ was added to $15 \mathrm{mg} / \mathrm{d}$ prednisolone. Further attempts to reduce steroid dose below $15 \mathrm{mg} / \mathrm{d}$ under maintenance therapy were accompanied by elevated inflammatory indices. Additionally, aggressive antihypertensive therapy consisting of enalapril, amlodipine, and diuretic, along with aspirin and osteoporosis prophylaxis, was commenced.

Six weeks later, the patient presented to her local hospital with acute visual problems. Neurological assessment confirmed a left-sided homonymous hemianopia and motor dysphasia. Intracerebral haemorrhage was excluded. Given simultaneous deterioration in CRP, a vasculitic exacerbation was considered the most likely explanation and high-dose steroids $(1000 \mathrm{mg} / \mathrm{d}$ IV SDH for 3 days, thereafter a further oral taper commencing with $60 \mathrm{mg} / \mathrm{d}$ ) were administered. A rapid improvement in CRP and complete resolution of the neurological symptoms were observed. Given the advanced nature of the carotid involvement, an elective leftsided aortocarotid bypass was performed 1 month later. Directly after surgery, a right-sided hemiparesis and newonset global dysphasia were diagnosed. An emergency MR brain confirmed minor intracerebral bleeding in the brainstem, believed to have resulted from an acute hypertensive crisis in the perioperative phase. Following multidisciplinary discussion, best supportive care was initiated including 12 weeks of intensive physiotherapy in a rehabilitation centre.

Despite ongoing maintenance treatment with azathioprine $150 \mathrm{mg} / \mathrm{d}$ and prednisolone $30 \mathrm{mg} / \mathrm{d}$, the patient developed dyspnoea and angina pectoris on exertion (NYHA 3), leading to transfer back to our care from the rehabilitation centre. Repeat MR staging excluded coronary artery involvement but high-grade stenosis of the right pulmonary artery was observed. Following review by colleagues in 


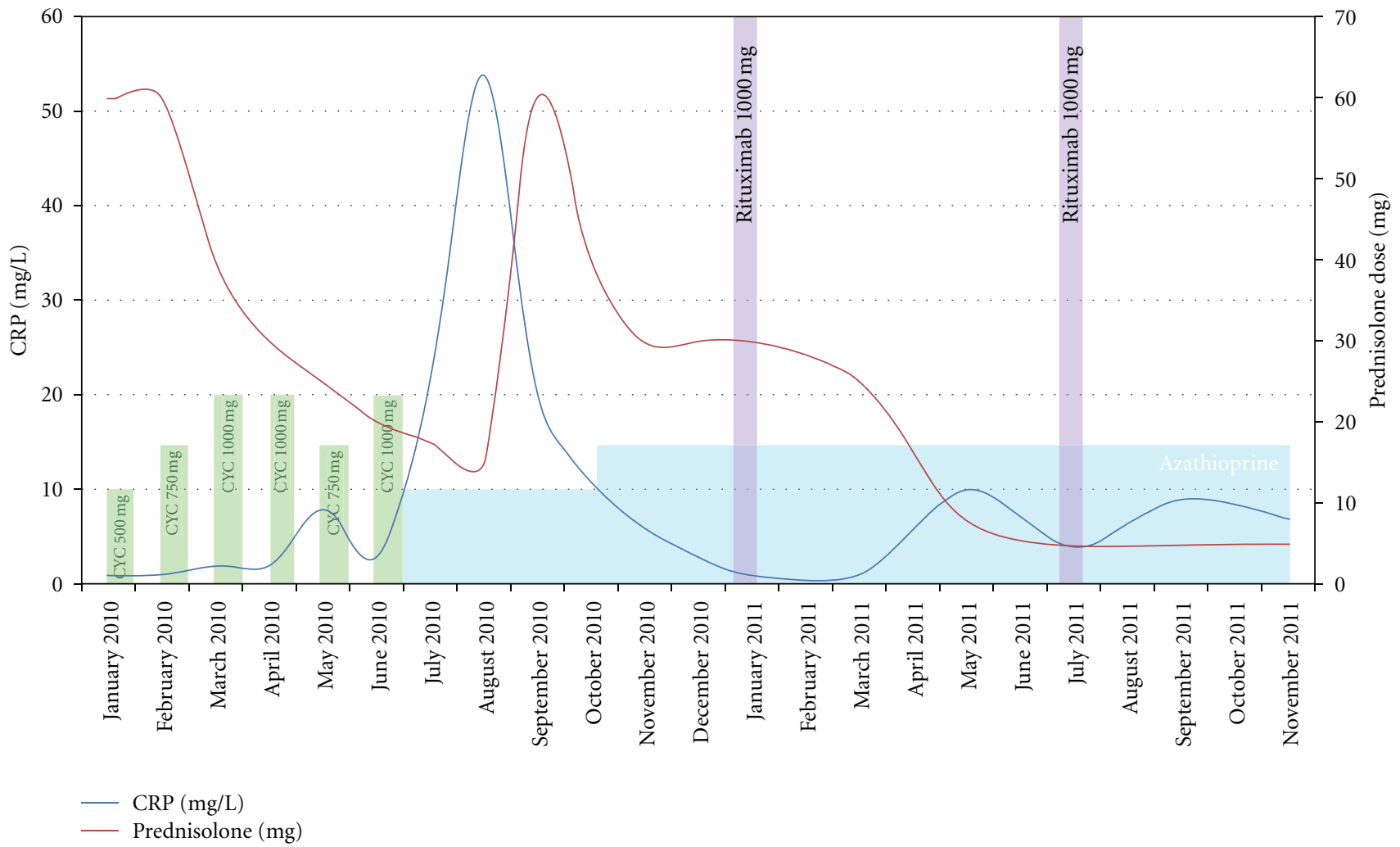

FIGURE 2: Treatment timeline showing the response of systemic inflammatory markers to different immunosuppressive therapies during the course of treatment. CRP: C-reactive protein; CYC: cyclophosphamide.

thoracic surgery, the patient's perioperative risk was deemed unacceptably high due to her poor clinical condition.

Given the failure to achieve satisfactory disease remission despite aggressive immunosuppression and in the absence of other established treatment modalities, the current literature was reviewed and discussed with the patient. Given emerging evidence for B-cell involvement and the efficacy of rituximab [3], this medication was selected as adjuvant therapy and administered per standard rheumatoid arthritis protocol: $1000 \mathrm{mg}$ on D1 and D15, repeated in 6 monthly intervals. Four weeks after the first cycle, the prednisolone dose was successfully reduced. Proceeding in $5 \mathrm{mg} /$ month dose reductions, no evidence of relapse was observed. For the past 12 months, the patient has remained in clinical remission with a maintenance dose of $5 \mathrm{mg} / \mathrm{d}$. Along with the stable inflammatory indices, there has been a steady improvement in clinical and functional statuses: full resolution of the neurological deficit, minimal exertional symptoms: NYHA 2, 3 flights of stairs without a pause, and a return to fulltime employment. Repeat MR staging 14 months after commencing rituximab confirmed that there was no indication of active vasculitis and that no further stenoses had occurred.

\section{Discussion}

Our case demonstrates a further example of rituximabinduced sustained remission in Takayasu arteritis, refractory to standard therapy. Unequivocal supporting evidence was demonstrated in terms of CRP and steroid requirement, stabilisation of the MR findings, and a remarkable improvement in both clinical and functional statuses. It once again highlights the dilemma of treating refractory TA, a problem that previous studies have identified in approximately $23 \%$ of patients [4]. In recent months, case reports and small retrospective studies have emerged proposing various biological therapies currently licensed for other rheumatological diseases as possible treatment options $[3,5,6]$.

Given the good correlation between CRP and clinical course in this patient, we were reluctant to sacrifice this through the use of the IL-6 receptor antagonist tocilizumab [6]. The most data clearly exists for infliximab [6]. We however preferred to use rituximab in our patient for the following two reasons.

(1) There was accompanying immunological data implicating B-cell involvement in active TA reported by Hoyer et al. [3]. They demonstrated raised plasmablast levels, leading to increased expression of CD19/CD20/CD27high/HLA-DR ${ }^{+}$B cells in peripheral blood during acute exacerbations [3].

(2) Using protein macroarrays and later on ELISA, we identified autoantibodies against ferritin in the majority of our TA patients (manuscript submitted) including this patient and in almost all our patients with active giant cell arteritis [7]. The presence of these autoantibodies suggests a role for B cells in TA. 
Our experience in this case added further evidence to a possible role for biological therapies in TA. Given the rare nature of the disease, numbers treated in individual centres will remain small, making meaningful prospective randomised studies difficult. We therefore propose the establishment of a registry of such patients, incorporating standardised disease staging, markers of activity and functional status to help guide future management in these patients.

\section{Acknowledgment}

The authors thank KFO 250 TP 03 for financial support.

\section{References}

[1] W. P. Arend, B. A. Michel, D. A. Bloch et al., "The American College of Rheumatology 1990 criteria for the classification of Takayasu arteritis," Arthritis and Rheumatism, vol. 33, no. 8, pp. 1129-1134, 1990.

[2] C. Mukhtyar, L. Guillevin, M. C. Cid et al., "EULAR recommendations for the management of large vessel vasculitis," Annals of the Rheumatic Diseases, vol. 68, no. 3, pp. 318-323, 2009.

[3] B. Hoyer, I. Mumatz, K. Loddenkemper, A. Bruns, C. Sengler, and K. Hermann, "Takayasu arteritis is charachterised by disturbances of B-cell homeostasis and responds to B-Cell depletion with rituximab," Annals of the Rheumatic Diseases, vol. 71, pp. 71-79, 2012.

[4] G. S. Kerr, C. W. Hallahan, J. Giordano et al., "Takayasu arteritis," Annals of Internal Medicine, vol. 120, no. 11, pp. 919-929, 1994.

[5] C. Salvarani, L. Magnani, M. Catanoso, N. Pipitone, A. Versari, and L. Dardani, "Tocilizumab: a novel therapy for patients with large-vessel vasculitis,” Rheumatology, vol. 51, pp. 51-56, 2012.

[6] A. Mekinian, A. Néel, J. Sibilia, P. Cohen, J. Connault, and M. Lambert, "Efficacy and tolerance of infliximab in refractory Takayasu arteritis: French multicentre study," Rheumatology, vol. 51, pp. 882-886, 2012.

[7] N. T. Baerlecken, A. Linnemann, W. L. Gross et al., "Association of ferritin autoantibodies with giant cell arteritis/polymyalgia rheumatica," Annals of the Rheumatic Diseases, no. 71, pp. 943 $947,2012$. 


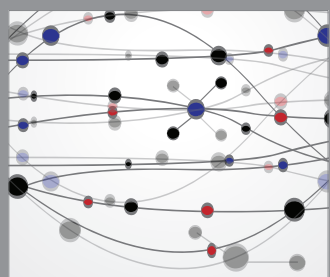

The Scientific World Journal
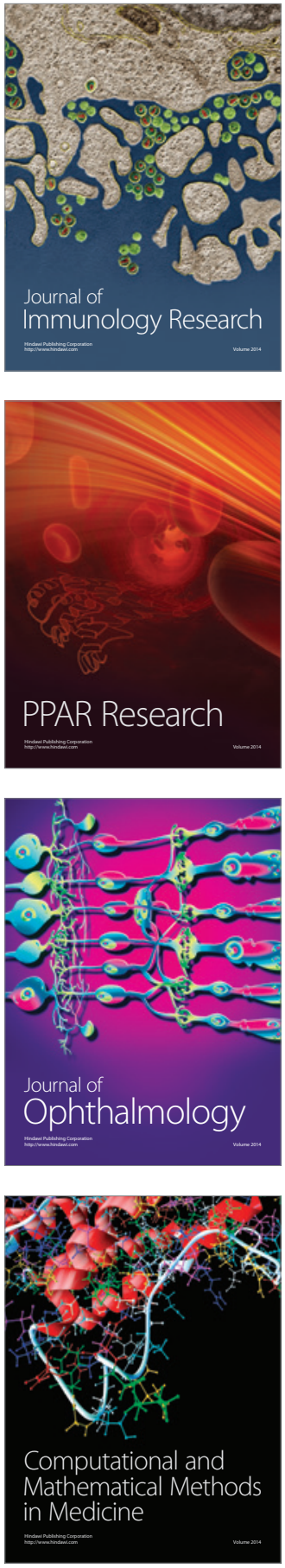

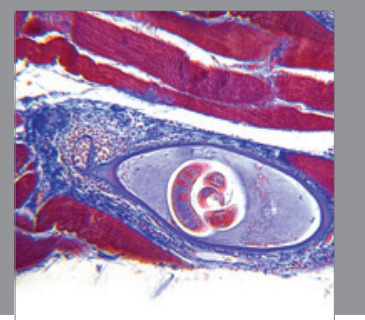

Gastroenterology

Research and Practice
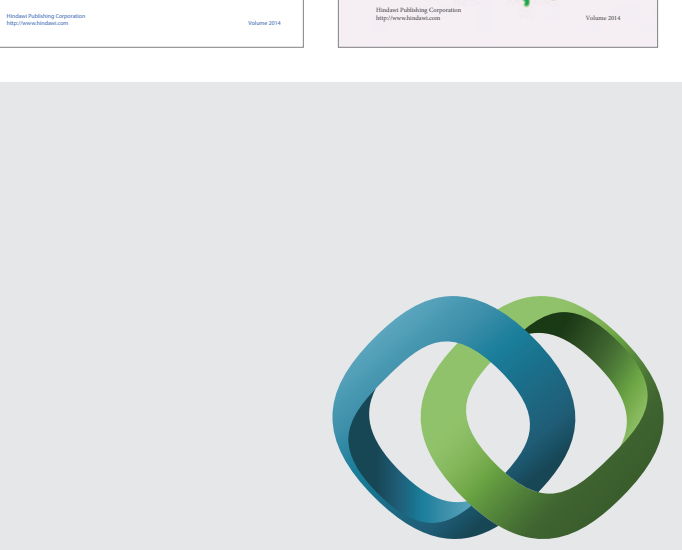

\section{Hindawi}

Submit your manuscripts at

http://www.hindawi.com
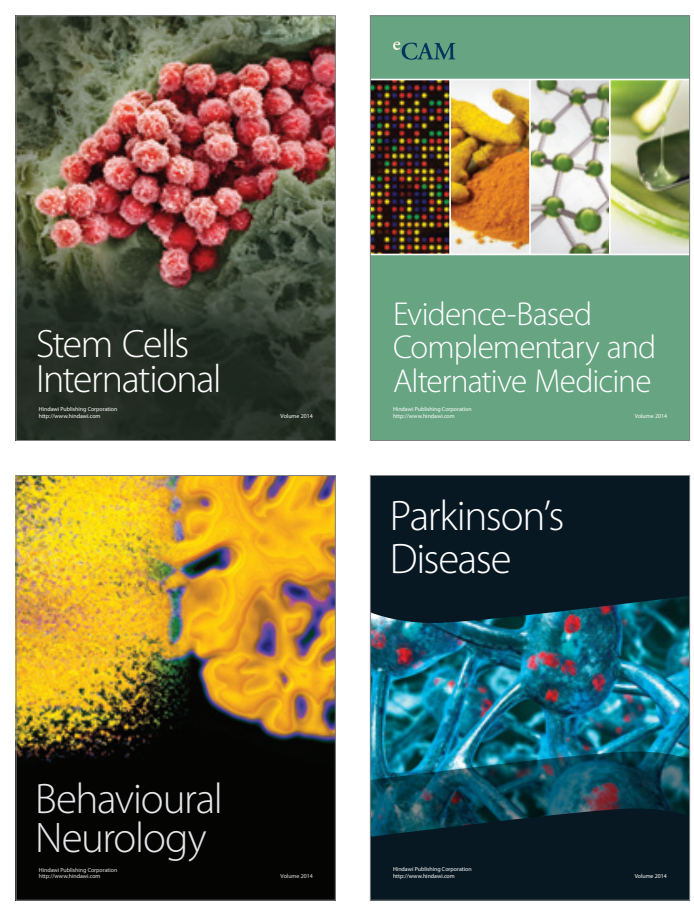

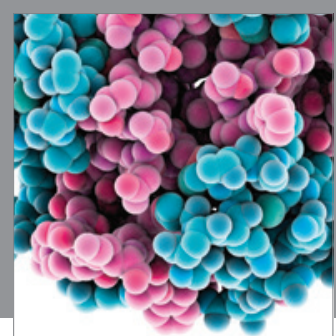

Journal of
Diabetes Research

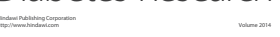

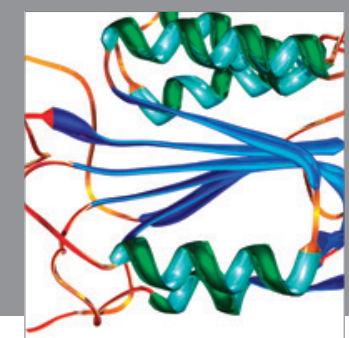

Disease Markers
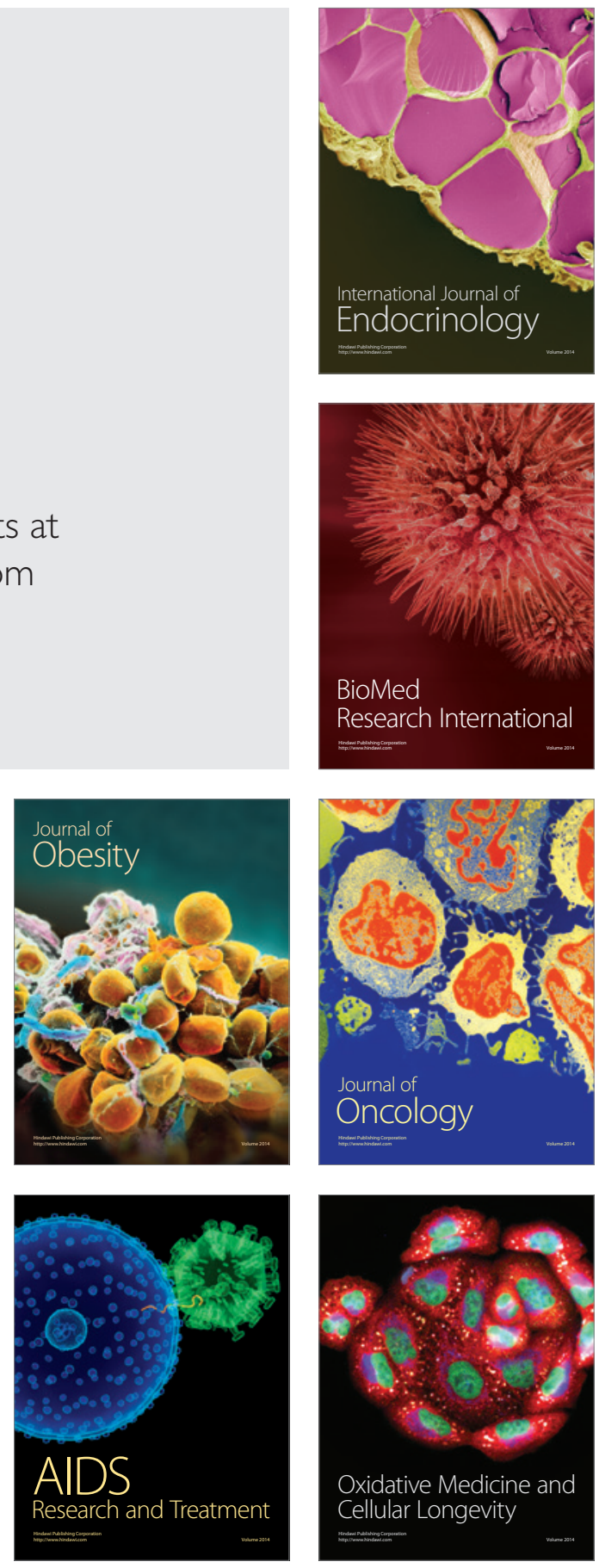